\title{
Letter
}

\section{Ligand-Conjugated Quantum Dots Monitor Antigen Uptake and Processing by Dendritic Cells}

Alessandra Cambi, Diane S. Lidke, Donna J. Arndt-Jovin, Carl G. Figdor, and Thomas M. Jovin Nano Lett., 2007, 7 (4), 970-977• DOI: 10.1021/nl0700503 • Publication Date (Web): 28 March 2007

Downloaded from http://pubs.acs.org on March 24, 2009

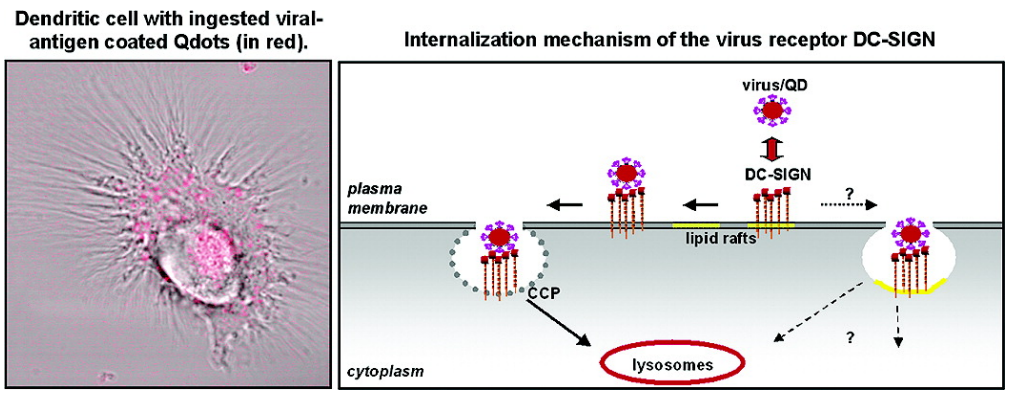

\section{More About This Article}

Additional resources and features associated with this article are available within the HTML version:

- Supporting Information

- $\quad$ Links to the 5 articles that cite this article, as of the time of this article download

- Access to high resolution figures

- $\quad$ Links to articles and content related to this article

- $\quad$ Copyright permission to reproduce figures and/or text from this article

View the Full Text HTML

\section{ACS Publications}




\title{
Ligand-Conjugated Quantum Dots Monitor Antigen Uptake and Processing by Dendritic Cells
}

\author{
Alessandra Cambi, ${ }^{*, t, \neq}$ Diane S. Lidke, ${ }^{\dagger, \S}$ Donna J. Arndt-Jovin, ${ }^{\dagger}$ \\ Carl G. Figdor, ${ }^{\ddagger}$ and Thomas M. Jovin ${ }^{\star}, \dagger$ \\ Department of Molecular Biology, Max Planck Institute for Biophysical Chemistry, \\ Göttingen, Germany, and Department of Tumor Immunology, Nijmegen Centre for \\ Molecular Life Sciences, Radboud University Nijmegen Medical Centre, \\ Nijmegen, The Netherlands
}

Received January 9, 2007; Revised Manuscript Received March 13, 2007

\begin{abstract}
The dendritic cell (DC) specific pathogen-uptake receptor (DC-SIGN) internalizes antigens for degradation and presentation onto MHC molecules. At the cell membrane, DC-SIGN forms nanoclusters that facilitate virus capture. However, internalized viruses, such as HIV-1, escape degradation. Here, we exploit ligand-conjugated, virus-sized, highly photostable quantum dots (QDs) to monitor in living cells antigen binding, entry, and trafficking. The antigen-coated QDs specific uptake and persistence in live DCs open the possibility for tracking antigen-presenting cells in vivo.
\end{abstract}

Dendritic cells (DCs) are phagocytic cells of hematopoietic origin located strategically for antigen $(\mathrm{Ag})$ sampling in tissues such as the mucosae that interface with the external environment. ${ }^{1}$ Upon Ag recognition, DCs are activated and migrate to the lymph nodes, where they present Ag-derived peptides to naive $\mathrm{T}$ lymphocytes and induce an effective immune response. The number of DCs circulating in the blood is extremely low; DCs are thus commonly generated in vitro by differentiation from monocyte precursors. ${ }^{2}$ Since DCs can be manipulated ex vivo, numerous efforts have been undertaken for exploiting them in the development of antitumor and antiviral therapies. ${ }^{3-5}$

Genomic approaches have been used to characterize DCs in more molecular terms. A series of genes have been identified, some DC specific, that encode lectin or lectinlike receptors. Many of these lectins are members of the calcium-dependent C-type lectin receptor (CLR) family and operate as constituents of the powerful Ag capture and uptake mechanism of DCs. ${ }^{6}$ Membrane-bound CLRs bind patho-

* Corresponding authors: T. M. Jovin, Department of Molecular Biology, Max Planck Institute for Biophysical Chemistry; tel, +49 551201 1382; fax, +49 551201 1467; e-mail, tjovin@gwdg.de. Alessandra Cambi, Department of Tumor Immunology, Nijmegen Centre for Molecular Life Sciences, Radboud University Nijmegen Medical Centre; tel, +31 24 17600; fax, +3124 3540339; e-mail, a.cambi@ncmls.ru.nl.

$\dagger$ Department of Molecular Biology, Max Planck Institute for Biophysical Chemistry.

$\doteqdot$ Department of Tumor Immunology, Nijmegen Centre for Molecular Life Sciences.

$\S$ Present address: Department of Pathology and Cancer Research and Treatment Center, University of New Mexico, Albuquerque, NM 87131. gens, which are then subjected to intracellular degradation and subsequent antigen loading of presenting MHC molecules responsible for the activation of $\mathrm{T}$ cells. ${ }^{7}$

Dendritic cell-specific ICAM-3-grabbing nonintegrin (DCSIGN; CD209) is a CLR initially described as an ICAM-3 (intercellular adhesion molecule-3) binding protein mediating DC $-\mathrm{T}$ cell interactions ${ }^{8}$ as well as a major HIV-1 receptor on DCs. ${ }^{9}$ DC-SIGN is responsible for the binding and uptake of several other pathogens, such as Ebola virus, ${ }^{10}$ hepatitis $\mathrm{C}$ virus (HCV), ${ }^{11}$ Candida albicans, ${ }^{12}$ and Mycobacterium tuberculosis. ${ }^{13}$ The interactions with these microorganisms occur upon recognition of a specific pathogen-associated carbohydrate moiety by DC-SIGN, either branched mannose structures (mannan) or fucose moieties (LewisX). ${ }^{14} \mathrm{Ag}$ uptake via DC-SIGN leads to Ag processing and presentation. ${ }^{7,15}$ However, pathogens such as HIV-1, HCV, and $M$. tuberculosis escape lysosomal degradation, thereby avoiding host immune surveillance. ${ }^{9,11,13,16}$

Despite the central role of DC-SIGN-mediated Ag uptake in DC function, little is known about the underlying molecular mechanism(s) of the initial entry and subsequent sorting pathways. Since neither the kinetics nor the uptake of ligands via DC-SIGN had been directly visualized, we undertook a study of the binding and internalization of "virussized" particles conjugated to carbohydrates or viral coat proteins. For this purpose, biotinylated ligands of DC-SIGN, such as LewisX or the HIV-1 envelope protein gp120, were bound to commercially available PEG-coated fluorescent 

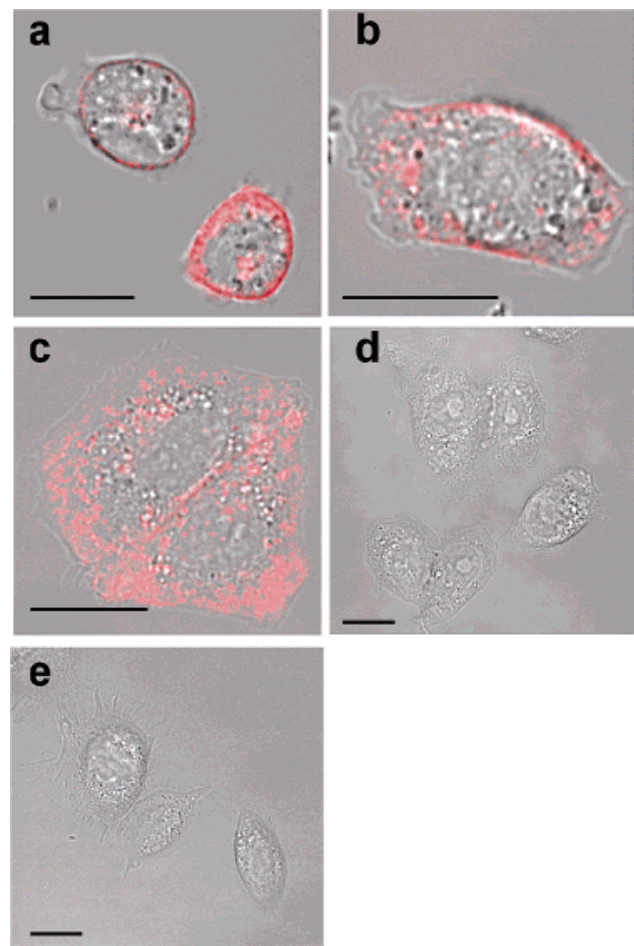

Figure 1. Ligand-coated QDs bind to DC-SIGN and are endocytosed into living cells: (a) AZN-D1-QDs; (b) gp120-QDs; (c) pLeX-QDs; (d) mLeX-QDs; (e) pLeX-QDs after preblocking in $50 \mathrm{mg} / \mathrm{mL}$ mannan. CHO cells stably transfected with DC-SIGN were incubated with $2 \mathrm{nM}$ ligand-conjugated QDs-605 for $30 \mathrm{~min}$ at $33{ }^{\circ} \mathrm{C}$. Binding was observed by confocal microscopy. Scale bars represent $10 \mu \mathrm{m}$.

quantum dots (QDs) bioconjugated with streptavidin. ${ }^{17}$ After conjugation, the diameter of the QDs was $\sim 40 \mathrm{~nm}$, comparable to the size of some viruses such as HCV. ${ }^{18,19}$ Due to their resistance to metabolic degradation as well as their exceptional photostability, ${ }^{17,20}$ the binding and intracellular behavior of QDs conjugated to receptor ligands can be monitored over long periods of time as shown in our laboratory in studies of the EGF receptor. ${ }^{21,22}$

Here we report the delineation of the DC-SIGN-mediated endocytosis mechanism in a similar fashion.

Ligand-Coated QDs Bind to DC-SIGN and Are Internalized. To investigate the intracellular routing of antigen internalization, we preloaded streptavidin QDs with biotinylated polymeric LewisX (pLeX-QDs), the HIV-1 envelope glycoprotein gp120 (gp120-QDs), or the anti-DC-SIGN mAb AZN-D1 (D1-QDs) (Supporting Information Table 1). The specificity of binding was demonstrated by binding to $\mathrm{CHO}$ cells that express human DC-SIGN (CHO-DC-SIGN). Confocal microscopy imaging demonstrated that all DCSIGN ligands conjugated to QDs were able to interact with and be internalized by DC-SIGN (Figure $1 \mathrm{a}-\mathrm{c}$ ). Unlike pLeX, monomeric LewisX (mLeX) is not recognized by DCSIGN. ${ }^{23}$ Accordingly, mLeX-QDs did not bind to the cells (Figure 1d). Furthermore, a clear inhibition was exerted by the carbohydrate mannan on the interaction between $\mathrm{CHO}-$ DC-SIGN and gp120-QDs (Figure 1e). Similar mannanmediated inhibition was observed when pLeX-QDs or D1QDs were used (data not shown).
We quantified both ligand binding and internalization of the specific ligand-QD complexes. Figure 2a shows a time course of live CHO-DC-SIGN cells incubated with pLeXQDs. Image segmentation processing was applied to define the membrane-associated- and the internalized-QD fraction (Figure $2 \mathrm{~b}$ inset). For quantitation, CHO-DC-SIGN cells were cotransfected with the cell surface receptor erbB1-EGFP (localized at the cell surface only) to delineate the plasma membrane during image segmentation. The presence of erbB1-EGFP did not alter the specific binding of ligandcoated QDs to DC-SIGN (Figure S1).

The kinetics of accumulation of pLeX-QDs at the cell surface and in the cytoplasm is shown in Figure $2 b$. Significant cell surface binding was visualized after 5-10 min, whereas internalization was detected only after $10-15$ min postaddition of QDs. Moreover, while the endocytosis followed a linear course, surface binding was more rapid and reached a plateau after $30 \mathrm{~min}$. Notably, no colocalization between internalized QDs and erbB1-EGFP was detected, indicating the absence of unspecific interactions between this receptor and the DC-SIGN-specific ligand-conjugated QDs. Similar results were obtained with gp120-QDs.

We compared these data with the binding and internalization of ligand-conjugated QDs by a DC-SIGN mutant, DCSIGN- $\Delta$ Repeat (Figure S2a), which lacks the extracellular neck domain thought to be responsible for tetramerization and is unable to bind to virus particles. ${ }^{24}$ The interaction of live CHO-DC-SIGN- $\Delta$ Repeat cells with pLeX-QDs was imaged under the same conditions described above for wildtype DC-SIGN (Figure 2b). Whereas binding and internalization of pLeX-QDs by wild-type DC-SIGN could be monitored for longer than $30 \mathrm{~min}$, the $\Delta$ Repeat mutant showed neither detectable binding nor internalization over the same period of time (Figure 2b and Figure S2b). Similar results were obtained when gp120-QDs were used with DCSIGN- $\Delta$ Repeat expressing cells (data not shown). The expression levels of DC-SIGN wild-type and $\Delta$ Repeat were comparable; thus, the difference in binding was not due to a smaller amount of the mutated DC-SIGN at the cell surface (data not shown).

We previously demonstrated that DC-SIGN can reside in cholesterol-enriched lipid rafts and that its binding properties are affected by perturbation of lipid microdomains (rafts). ${ }^{25}$ Disruption of lipid rafts by adding the cholesterol extracting agent methyl- $\beta$-cyclodextrin (MCD) to CHO-DC-SIGN cells significantly decreased the binding rate and greatly inhibited the internalization of pLeX-QDs (Figure $2 b$ and Figure S2c). Moreover, even after $60 \mathrm{~min}$ of incubation with the QDs, only a weak binding was observed at the plasma membrane of the treated cells and there was no internalization (Figure S2d). These results demonstrate that only QDs conjugated to specific ligands are recognized and internalized by DC-SIGN and that the oligomerization state of DC-SIGN as well as membrane raft integrity greatly influenced the interaction with these fluorescent, virus-sized particles.

Time-Dependent Colocalization of Internalized QDs with Different Cellular Compartments. We used gp120QDs to elucidate the entry steps of DC-SIGN-mediated 

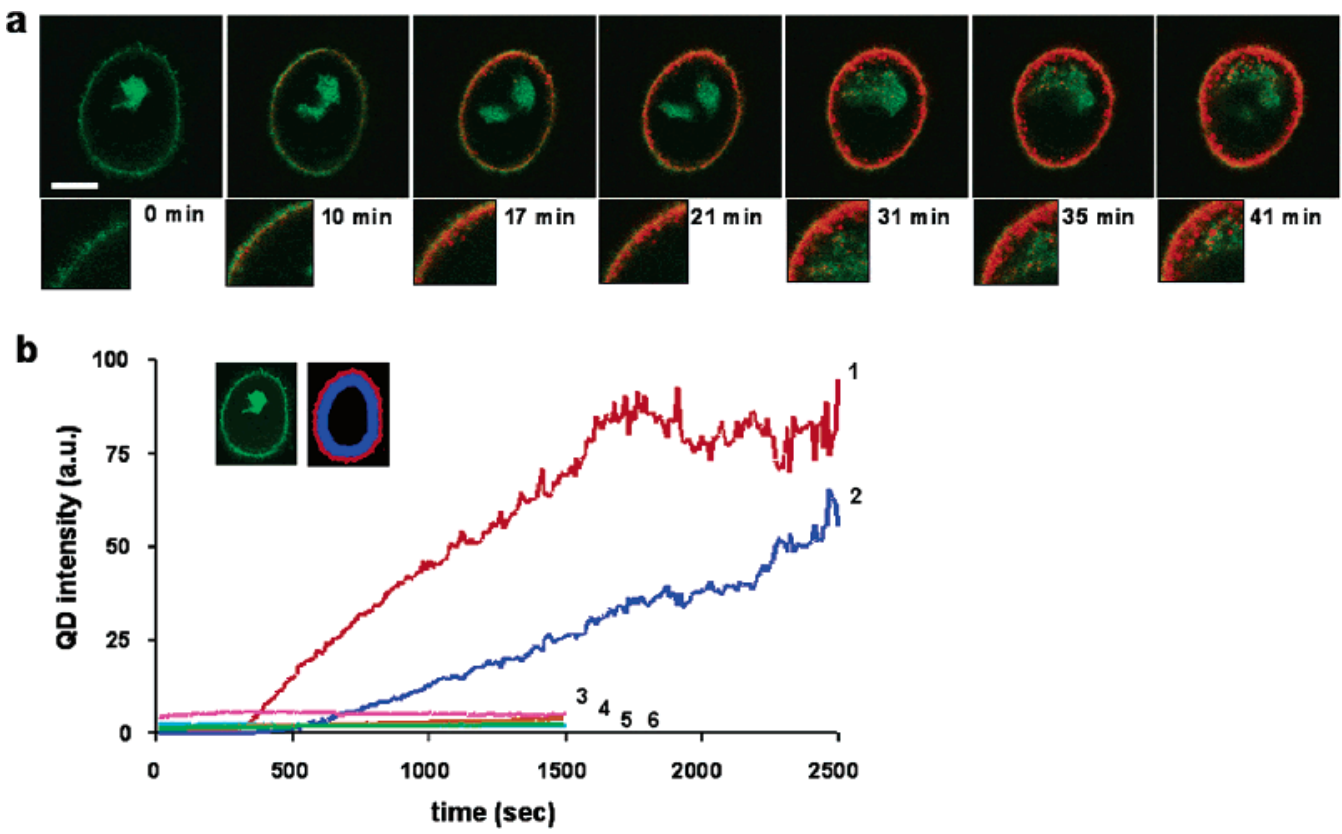

Figure 2. Quantitative analysis of real-time binding and internalization of ligand-coated QDs. (a) Time series of CHO-DC-SIGN cells incubated with $2 \mathrm{nM}$ gp120-QD655. Cotransfection of erbB1-EGFP labeled the cell membrane and allows delineation from cytoplasm. (b) Comparative kinetics of binding and internalization for CHO-DC-SIGN. Inset: cellular image (left) and masks (right) corresponding to cell surface (red) and internal regions of interest (blue, defined automatically during analysis; see supplementary methods). Plot shows total gp120-QDs signal on the cell membrane (red, 1) or inside the cell (blue, 2) as a function of time for CHO-DC-SIGN wild-type; binding (pink, 3) and internalization (light blue, 4) of gp120-QDs in time for CHO-DC-SIGN- $\Delta$ Repeat; binding (orange, 5) and internalization (green, 6) of QDs on CHO-DC-SIGN after 15 min preincubation in $20 \mathrm{mM} \mathrm{MCD}$. Scale bar represents $2.5 \mu \mathrm{m}$.

antigen uptake and the subsequent endocytic pathway by establishing the degree of colocalization of the QD signals with known markers of cellular compartments. CHO-DCSIGN cells and gp120-coated QDs were incubated for different periods of time (Figure 3). After fixation and permeabilization, $\mathrm{CHO}-\mathrm{DC}$-SIGN cells were labeled with fluorescent $\mathrm{mAb}$ specific for different markers of the endocytic pathway and analyzed by confocal microscopy. A significant number of QDs colocalized with either DCSIGN-enriched vesicles or clathrin-coated pits (CCPs) (Figure $3 \mathrm{a}, \mathrm{b}$ ) after binding and internalization for $10 \mathrm{~min}$, but the correlation was lost at longer times of incubation (Figure 3d,e). In contrast to clathrin, QDs failed to colocalize with caveolin-1 at either early or late time points (Figure 3c,f). We quantified the colocalization of the internalized QDs with DC-SIGN, clathrin, or caveolin-1 by calculating the correlation coefficient $\mathrm{C}$ (see methods) in multiple images. As shown in Figure 3g, only DC-SIGN and clathrin showed a significant colocalization at early time points, which was lost after prolonged incubation. Further support for the notion that DC-SIGN-mediated endocytosis of gp120-QDs is clathrin-dependent was provided by the colocalization of internalized gp120-QDs with mRFP-Rab5 in CHO-DC-SIGN cells (Figure 3h). Rab5 is present in vesicles that have just shed their clathrin coats and are entering the early endosomal compartment. ${ }^{26}$ Internalized QDs never colocalized with the Golgi apparatus, as shown by labeling with the Golgi marker SulfoT-EGFP (Figure 3i). Similar results were obtained with pLeX-QD (data not shown). These data clearly demonstrate that DC-SIGN-mediated endocytosis of virus-sized particles occurs via a clathrin-dependent, caveolin-independent pathway.

Dendritic Cells Bind and Internalize Ligand-Coated QDs. Although DC-SIGN expressed in CHO cells served in this paper and previous work ${ }^{27}$ as a valid model system for studying the CLR, these cells do not recapitulate the full repertoire of activities of native DCs. Thus, we performed similar experiments with ligand-conjugated QDs on DCs. Binding of gp120-QDs to fresh human DCs was detectable 10 min after addition of the QDs (Figure 4a) and internalization increased thereafter. Prior incubation of DCs with the blocking anti-DC-SIGN mAb AZN-D1 specifically prevented binding of the gp120-QDs to DCs (Figure 4b). Moreover, no unspecific binding was observed upon addition of ligandunconjugated streptavidin-QDs (data not shown). Figure 4c shows a reconstructed three-dimensional image of DCs that had internalized many gp120-QDs during $30 \mathrm{~min}$ of incubation. Subsequent to fixation, DC-SIGN was labeled extracellularly with a mAb in order to define the cell membrane. Interestingly, most of the endocytosed QDs accumulated at the center of the cell, although a large fraction was still visible in smaller structures underneath the cell membrane. The orthogonal sections of another DC (Figure 4d) further support the conclusion that the DCs internalized the ligandconjugated QDs.

GP120-QDs Reach the Antigen Processing and Loading Compartment. To further investigate the intracellular destiny of the internalized QDs on DCs, we labeled several molecules potentially involved in the endocytic pathway. Similarly to CHO-DC-SIGN cells, DCs also internalized 

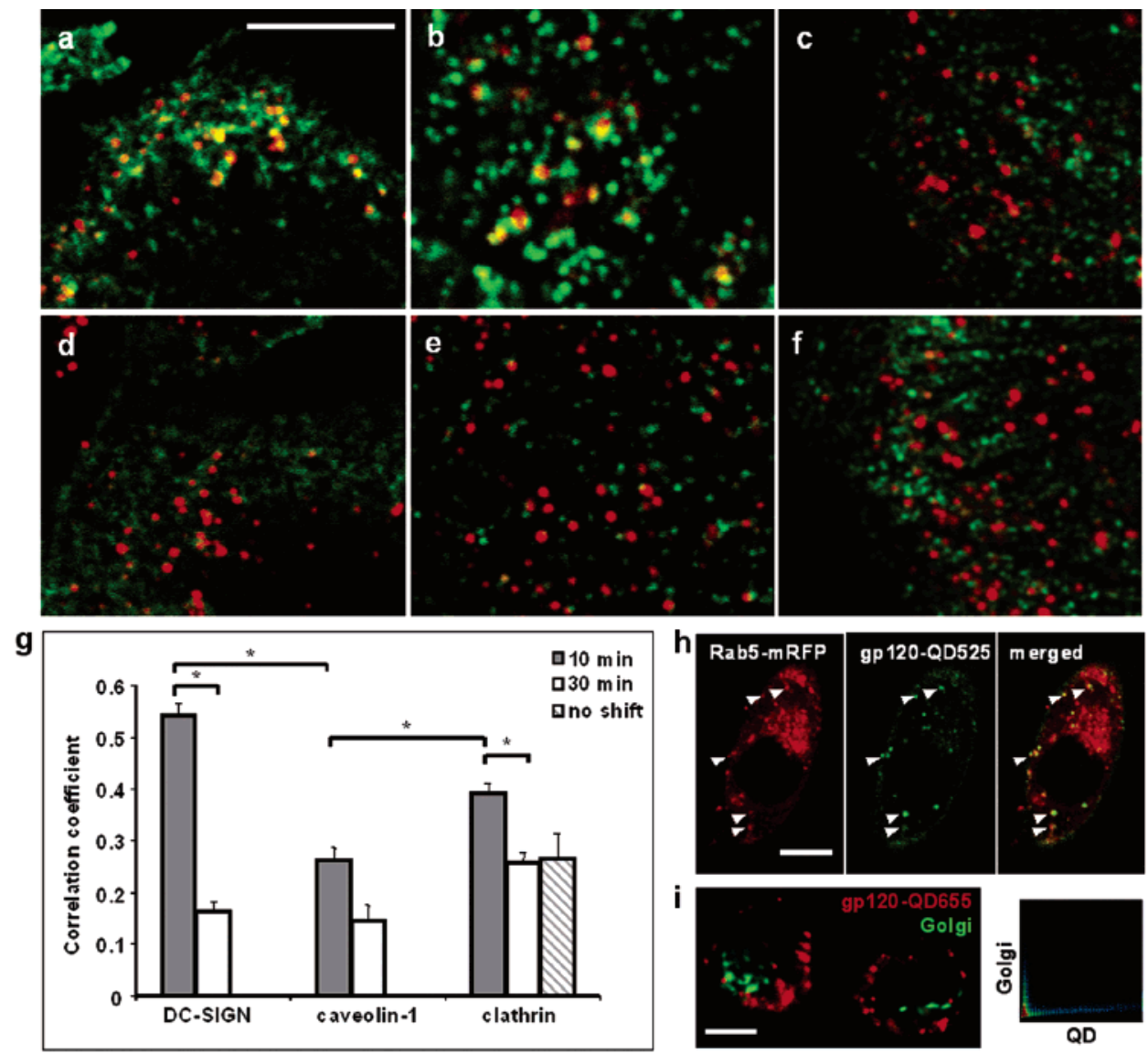

Figure 3. Ligand-coated QDs are internalized via DC-SIGN and colocalize with CCP. CHO-DC-SIGN cells were incubated with $20 \mathrm{nM}$ pLeX-QD655 for $30 \mathrm{~min}$ at $12{ }^{\circ} \mathrm{C}$ to allow binding but prevent internalization. A subsequent temperature shift at $37{ }^{\circ} \mathrm{C}$ for $10 \mathrm{~min}(\mathrm{a}-\mathrm{c})$ or $30 \mathrm{~min}(\mathrm{~d}-\mathrm{f})$ induced simultaneous internalization of the bound QDs (red). After thorough washing, fixation in $4 \%$ PFA, and permeabilization in Triton X-100, the various markers (green) were labeled with specific primary mAb and fluorescent conjugated secondary Ab (a,d DC-SIGN; b,e clathrin; c,f caveolin-1). (g) Plot showing the correlation coefficient $C$ (see methods) between the internalized QDs and the indicated markers (no shift bar indicates colocalization between QDs and clathrin in absence of temperature shift at $37{ }^{\circ} \mathrm{C}$ for 10 min). Values \pm SEM are average of multiple images from several cells in two independent experiments $(*=P<0.001, T$ test). (h) CHO-DC-SIGN cells were transiently transfected with Rab5-mRFP (red) and incubated with 2 nM gp120-QD525 (green). Single optical section after $15 \mathrm{~min}$ from addition of the QDs. (i) CHO-DC-SIGN were transiently transfected with SulfoT-EGFP (green) and incubated with $2 \mathrm{nM}$ gp120-QD655 (red). Single optical section after $30 \mathrm{~min}$ from addition of the QDs. Right panel represents a two-dimensional histogram of EGFP signal vs gp120-QD655 signal: the totally nonoverlapping dots indicate total lack of colocalization. Scale bars represent $5 \mu \mathrm{m}$.

GP120-QDs via DC-SIGN-enriched vesicles (Figure 5a) and via CCP (Figure 5b). No colocalization between QDs and caveolin or the integrin LFA-1 was observed (Figure S3). In addition, we determined that at incubation times longer than 30 min the QD accumulated in the lysosomal compartment, as shown by the strong colocalization of the internalized QD with the lysosomal marker LAMP-1 (Figure 5c). Moreover, under the same conditions, an anti-gp120 mAb revealed that the coated ligand (i.e., gp120) completely colocalized with the QDs both in early endosomes and in lysosomes (Figure 5d). The specificity of this labeling was confirmed by the absence of gp120 signal in the neighboring DCs that had not internalized the QDs.

Finally, we investigated whether the internalized QDs could be detected inside the DC after prolonged incubations. The DCs were exposed to gp120-QDs for $1 \mathrm{~h}$ at $37^{\circ} \mathrm{C}$. After the excess of unbound QDs was washed away, the cells were kept in culture in normal medium for $48 \mathrm{~h}$ and imaged. The cargo of intracellular QDs (Figure S4) was clearly evident and was demonstrated to be colocalized with the MHCII enriched compartment, identified with a marker for HLADR (Figure 5e).

Discussion. We previously reported the application of photostable QDs to study signal transduction. ${ }^{21,22}$ Here, we made use of ligand-conjugated QDs for visualizing the binding and internalization of antigens mediated by DCSIGN in living cells. We demonstrated that DC-SIGN internalizes virus-sized ligand-conjugated QDs via a clathrindependent mechanism. Colocalization of the internalized QDs with DC-SIGN and clathrin was lost $10 \mathrm{~min}$ after internalization, and at times longer than 30 min postbinding, the QDs, as well as the conjugated ligand, accumulated in the lysosomal compartment. Finally, we showed that internalized QDs could be detected inside living cells even after prolonged (up to $48 \mathrm{~h}$ ) incubations and strongly colocalized with MHCII-enriched compartments. These results suggest that internalization of Ags conjugated to QDs alters neither cell viability nor the proper $\mathrm{Ag}$ degradation inside lysosomes 


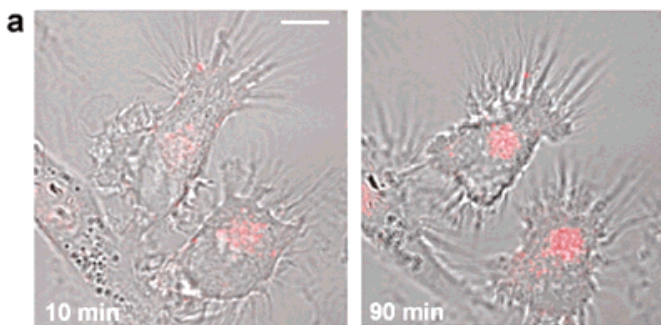

b

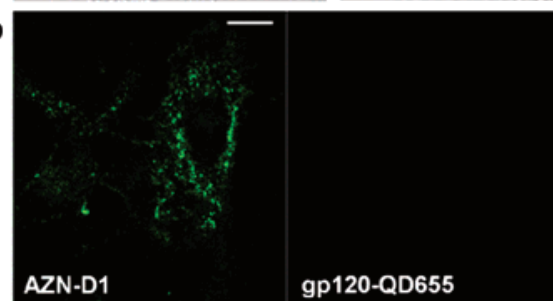

C

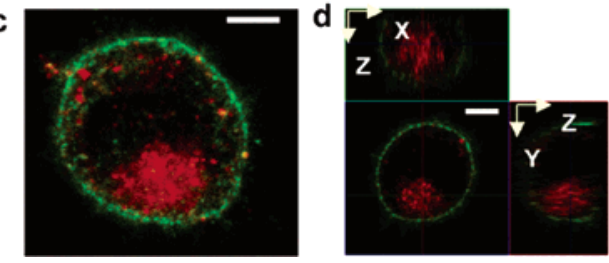

Figure 4. DCs bind and internalize ligand-coated QDs. DCs were incubated with $2 \mathrm{nM}$ gp120-QD605 at $33{ }^{\circ} \mathrm{C}$ and binding was imaged by confocal microscopy. (a) Single optical section at selected time points of DC after addition of QDs (red). (b) DCs were first incubated with the Alexa-488-conjugated anti-DC-SIGN blocking mAb AZN-D1 $(20 \mathrm{mg} / \mathrm{mL})$ for $10 \mathrm{~min}$ at $33{ }^{\circ} \mathrm{C}$, after which the QDs were added. (c) Stereo image and (d) orthogonal sections of DCs with internalized gp120-QD (red) and membrane DC-SIGN labeling (green) after 90 min incubation with QDs, washing, and fixation. Images are representative of multiple cells from several independent experiments. Scale bars represent $10 \mu \mathrm{m}$.

and subsequent loading of MHCII molecules. Thus, the QDs provide a new, sensitive, and convenient method for following antigen-loaded cells as discussed below.

The binding of the ligand-conjugated QDs to the cell surface could be visualized after 5-10 min, reaching a plateau after $30 \mathrm{~min}$, whereas the endocytosis followed a linear course subsequent to binding (Figure 2). We conclude that the cellular endocytotic machinery is the rate-limiting process for the internalization of ligand-conjugated QDs via DC-SIGN.

Previous studies from one of our laboratories revealed that the organization of DC-SIGN in discrete nanoclusters at the DC plasma membrane is essential for binding and uptake of virus particles and that both clustered and randomly distributed DC-SIGN molecules bind to micrometer-sized particles as well as soluble ligands. ${ }^{25}$ Here, we extend these findings and demonstrate that the mutant DC-SIGN- $\Delta$ Repeat, defective in forming tetramers and of binding to HIV $-1,{ }^{24}$ does not bind or internalize ligand-conjugated QDs (Figure $2 b$ and Figure S2). These observations confirm a similarity in size and antigenicity of the ligand-conjugated QDs used in our experiments compared with virus particles as well as the absence of large cross-linked QD aggregates.

Using complementary techniques, we and others showed that DC-SIGN may reside within lipid rafts, ${ }^{25,28}$ a finding consistent with the inhibition of QD-ligand binding and internalization of cholesterol-depleted CHO-DC-SIGN cells (Figure 2 and Figure S2). In the case of both CHO-DCSIGN and DCs, QD-ligands internalized through DC-SIGN colocalize with CCPs (Figure 3 and 5). CCPs and lipid rafts are considered to be mutually exclusive compartments of the plasma membrane. ${ }^{29}$ However, cholesterol extraction has been also reported to affect CCP formation..$^{30}$ Although a dileucine motif, which is presumed to mediate the interaction with CCPs,${ }^{31}$ has been identified in the cytoplasmic tail of DC-SIGN, ${ }^{7}$ direct evidence for an interaction between DCSIGN and clathrin has been lacking so far. Our study provides the first clear demonstration that the internalization of DC-SIGN antigens occurs via a clathrin-dependent, caveolin-independent mechanism (Figures $3 \mathrm{a}-\mathrm{g}$ and $5 \mathrm{~b}$ ). Conceivably, ligand binding induces DC-SIGN to exit lipid rafts and redistribute with its bound antigen toward CCPs (Figure 6). A raft-dependent, clathrin-independent mechanism may also play a role in DC-SIGN-mediated endocytosis (Figure 6). These possibilities are presently under investigation.

We quantified the colocalization between internalized ligand-conjugated QDs and DC-SIGN- or clathrin-enriched vesicles at different time points (Figure 3). Interestingly, 10 min after internalization the endocytosed QDs seemed to segregate from DC-SIGN, suggesting that the ligand and receptor followed different internalization routes. Whether DC-SIGN recycles back to the surface or is quickly degraded in the lysosomes is still unknown. The application of Agconjugated QDs in combination with compounds interfering with either exo- or endocytosis will hopefully provide additional information in this regard.

Direct in vivo visualization of cellular macromolecules has been successfully achieved in many systems by the introduction of transgenes expressing visible fluorescent protein fusions. We prepared and transfected a construct of GFP fused to the intracellular N-terminus of DC-SIGN into different cell lines (data not shown). This fusion protein, while capable of binding to micrometer-sized ligand-coated particles, ${ }^{32}$ did not bind to ligand-conjugated QDs as efficiently as the wild-type DC-SIGN (Figure S5). We analyzed CHO-DC-SIGN-GFP expressing cells by energy migration Förster resonance energy transfer (emFRET or homotransfer FRET) measured in the confocal laser-scanning microscope..$^{33}$ We found that the DC-SIGN-GFP on the cell surface was primarily present as a dimer (Lidke et al., unpublished data). Since protein tetramerization is required in order for DCSIGN to bind multivalent carbohydrates,$^{34}$ we conclude that the presence of GFP interferes with the formation of a proper quaternary structure, thereby hampering the virus binding capacity.

Standard techniques for transfecting DNA constructs into primary DCs are highly inefficient, thus limiting the application of visible fluorescent protein (VFP)-based constructs to study DC-specific receptors directly within their physiological context. Quantitative approaches based on multiple combinations of QDs and ligands provide an alternative means for simultaneously tracking the internalization of 

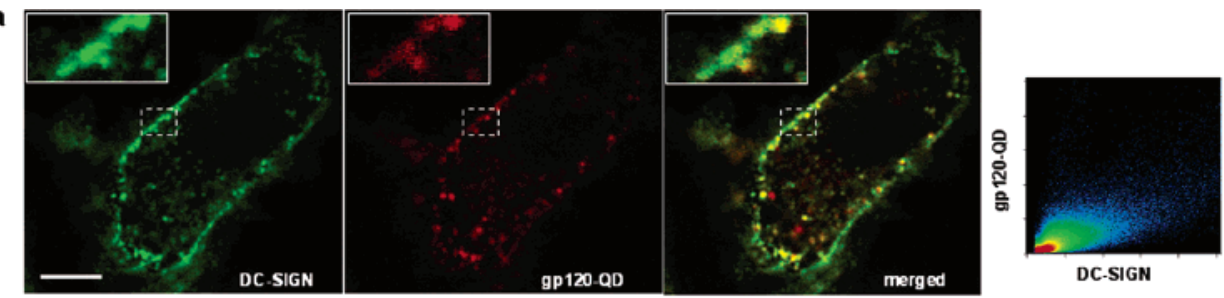

b
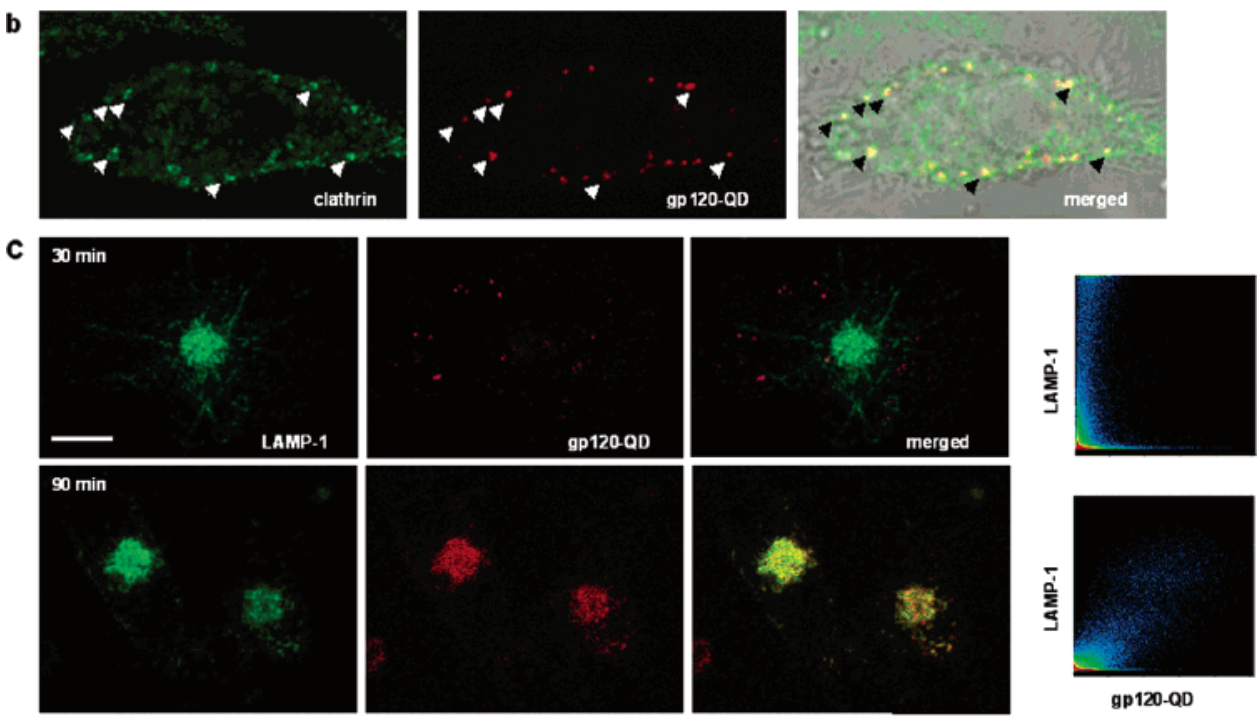

d
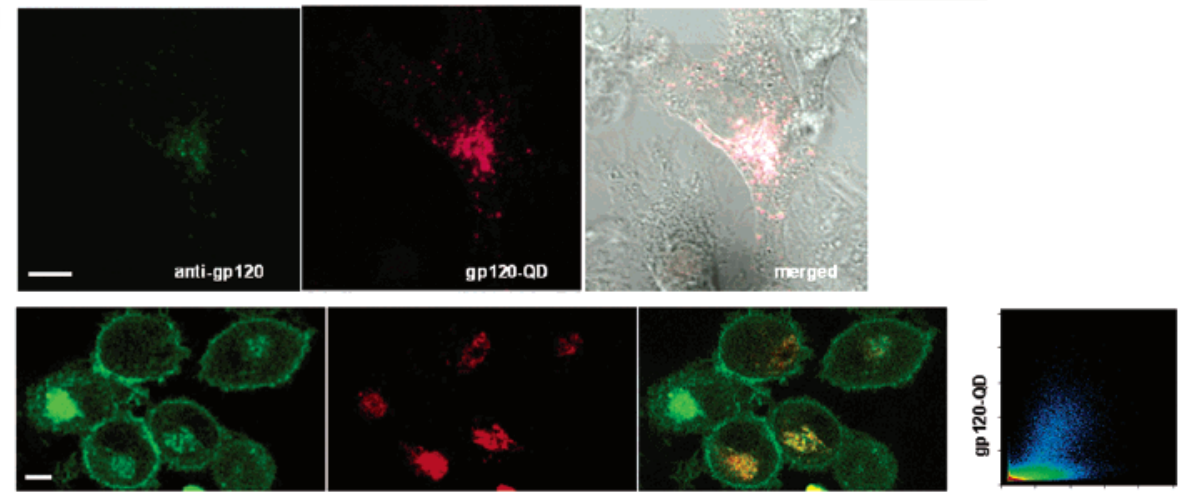

HLA.DR

Figure 5. Ligand-conjugated QDs internalized by the DCs via DC-SIGN and CCP reach the antigen processing and loading compartment. DCs were incubated with $2 \mathrm{nM}$ gp120-QD605 (red) at $33^{\circ} \mathrm{C}$ for up to $90 \mathrm{~min}$. After thorough washing of unbound QDs, DCs were fixed in $4 \%$ PFA and permeabilized, and the various markers (green) were labeled with specific primary mAb and fluorescent conjugated secondary $\mathrm{Ab}$ (see methods). Data were obtained by confocal microscopy. (a) DC-SIGN was labeled after 15 min of incubation with QDs. Inset: enlarged detail of the picture showing a DC-SIGN vesicle pinching off the cell membrane and containing QDs. Right panel represents a two-dimensional (2D) histogram of DC-SIGN signal vs gp120-QD605 signal: the small amount of dispersion indicates high colocalization. (b) Clathrin was labeled after 15 min of incubation with QDs. The arrows indicate some of the QDs colocalizing with CCP. (c) The lysosomal marker LAMP-1 was labeled after 30 and 90 min of incubation with QDs. Right panels, 2D histograms of LAMP-1 signal vs gp120-QD605 signal. The high dispersion in the upper 2D histogram (30 min) is indicative of little colocalization. The small amount of dispersion in the lower 2D histogram (90 min) indicates high colocalization. (d) gp120 conjugated to the QDs was labeled with an antigp120 mAb. (e) DCs were incubated with $2 \mathrm{nM}$ gp120-QD605 at $33{ }^{\circ} \mathrm{C}$ for up to $90 \mathrm{~min}$. After thorough washing of unbound QDs, DCs were then put back in their culture medium at $37{ }^{\circ} \mathrm{C}$ for $48 \mathrm{~h}$. Subsequently, DCs were fixed in $4 \%$ PFA, permeabilized, labeled with specific primary mAb and fluorescent conjugated secondary Ab (see methods), and analyzed by confocal microscopy. Single optical section of DCs with internalized QD (red) and labeled with anti-HLA-DR mAb (green) is shown. Right panel, 2D histograms of HLA-DR signal vs gp120-QD605 signal. Representative pictures out of several experiments are shown. Scale bars represent $10 \mu \mathrm{m}$.

different antigens via different receptors for prolonged periods of times.

Many studies have been directed at the exploitation of DCs to improve antiviral or antitumor vaccine efficiency (reviewed in ref 3). In the technique widely used in current clinical trials, DCs are loaded with antigens by ex vivo incubation with MHC class I and II binding peptides, followed by reintroduction of antigen-loaded DCs back into a patient to assess their T cell activation potential. ${ }^{3}$ Antigencoupled QDs ingested by DCs are a potential tool for determining the location and duration of therapeutic DCs. In this study we determined that internalized QDs, still bearing the ligand, colocalized with the LAMP-1-enriched compartment, showing that the ligand-conjugated QDs are 


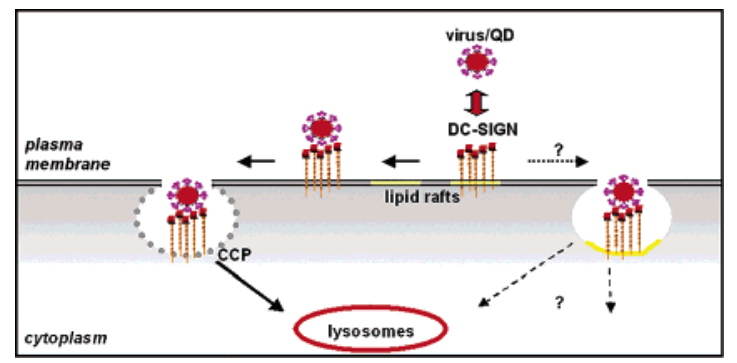

Figure 6. DC-SIGN internalization pathways. At the cell surface of DCs, DC-SIGN nanoclusters may reside within lipid rafts. ${ }^{25}$ Upon binding of virus or virus-sized ligand-conjugated QD, DC-SIGN nanoclusters might move out of lipid rafts and internalize via CCP.

not retained in vesicles of the early sorting pathways but are instead able to reach the lysosomes (Figure $5 \mathrm{c}, \mathrm{d}$ ). Moreover, the fluorescent signal of endocytosed QDs in DCs was still detectable for at least 2 days after internalization and colocalized with the MHCII loading compartment (Figure 5e and Figure S4). Being nontoxic, metabolically resistant, and highly photostable, QDs could serve to monitor antigen-loaded DC within tissues after readministration to animals.

Compared to exogenous peptide loading, a more direct and less laborious strategy for improving vaccine efficiency would be to target Ags to DCs in vivo via specific cell surface receptors. Recently, several studies conducted on antigen targeting to CLRs for vaccination purposes indicated that CLRs are potential Ag targeting receptors. ${ }^{4,35,36}$ In fact, antibody-mediated targeting of Ag to DCs via DC-SIGN effectively induced $\mathrm{Ag}$-specific naive as well as recall T-cell responses. ${ }^{15}$ On the other hand, DC-SIGN is targeted by pathogens like M. tuberculosis, HIV-1, and HCV, thereby avoiding degradation in lytic compartments. ${ }^{11,13,16}$ Although ligand-conjugated QDs do not exactly reproduce the immune escape of viruses, they constitute very useful tools for mimicking viral binding and entry. The more detailed information thereby gained on how different Ags are internalized via DC-SIGN and other CLRs will be instrumental for the rationale design of efficient CLR-based therapeutic strategies.

The broad range of QD colors and sizes allows simultaneous tracking of DCs loaded with different antigens or even different DC subsets. The recent advances in the chemistry of QD coating permit the simultaneous conjugation of two or more different molecules, and thus, the simultaneous stimulation of several receptors, ${ }^{37}$ further broadening the application potential of these fluorescent probes for dynamic studies of cellular processes.

Acknowledgment. The authors thank Ben Joosten and Inge Beeren for excellent technical assistance in the preparation of transfectants and isolation of monocytes and dendritic cell differentiation. This work was supported by an EMBO long-term fellowship (ALTF 263-2005) to A,C. A,C, also acknowledges the Netherlands Organization for Scientific Research (NWO Grant R93-481) and the Dutch Society of Immunology (NVvI). D.S.L. was the recipient of a postdoctoral fellowship from the EU FP5 Grant QLG2-CT-200002278 awarded to T.M.J.
Supporting Information Available: Experimental details including reagents, cell culture, cell staining, preparation of QDs, and confocal microscopy analysis as well as supplementary figures. This material is available free of charge via the Internet at http://pubs.acs.org.

\section{References}

(1) Banchereau, J.; Steinman, R. M. Nature 1998, 392 (6673), 245252.

(2) Romani, N.; Gruner, S.; Brang, D.; Kampgen, E.; Lenz, A.; Trockenbacher, B.; Konwalinka, G.; Fritsch, P. O.; Steinman, R. M.; Schuler, G. J. Exp. Med. 1994, 180 (1), 83-93.

(3) Figdor, C. G.; de Vries, I. J.; Lesterhuis, W. J.; Melief, C. J. Nat. Med. 2004, 10 (5), 475-480.

(4) Bonifaz, L. C.; Bonnyay, D. P.; Charalambous, A.; Darguste, D. I.; Fujii, S.; Soares, H.; Brimnes, M. K.; Moltedo, B.; Moran, T. M.; Steinman, R. M. J. Exp. Med. 2004, 199 (6), 815-824.

(5) Ludewig, B. Curr. Top. Microbiol. Immunol 2003, 276, 199-214

(6) Figdor, C. G.; van Kooyk, Y.; Adema, G. J. Nat. Rev. Immunol. 2002, 2 (2), 77-84.

(7) Engering, A.; Geijtenbeek, T. B.; van Vliet, S. J.; Wijers, M.; van Liempt, E.; Demaurex, N.; Lanzavecchia, A.; Fransen, J.; Figdor, C. G.; Piguet, V.; van Kooyk, Y. J. Immunol. 2002, 168 (5), 2118 2126

(8) Geijtenbeek, T. B.; Torensma, R.; van Vliet, S. J.; van Duijnhoven, G. C.; Adema, G. J.; van Kooyk, Y.; Figdor, C. G. Cell 2000, 100 (5), 575-585.

(9) Geijtenbeek, T. B.; Kwon, D. S.; Torensma, R.; van Vliet, S. J.; van Duijnhoven, G. C.; Middel, J.; Cornelissen, I. L.; Nottet, H. S. KewalRamani, V. N.; Littman, D. R.; Figdor, C. G.; van Kooyk, Y. Cell 2000, 100 (5), 587-597.

(10) Alvarez, C. P.; Lasala, F.; Carrillo, J.; Muniz, O.; Corbi, A. L.; Delgado, R. J. Virol. 2002, 76 (13), 6841-6844.

(11) Ludwig, I. S.; Lekkerkerker, A. N.; Depla, E.; Bosman, F.; Musters, R. J.; Depraetere, S.; van Kooyk, Y.; Geijtenbeek, T. B. J. Virol. 2004, 78 (15), 8322-8332.

(12) Cambi, A.; Gijzen, K.; de Vries, J. M.; Torensma, R.; Joosten, B.; Adema, G. J.; Netea, M. G.; Kullberg, B. J.; Romani, L.; Figdor, C. G. Eur. J. Immunol. 2003, 33 (2), 532-538.

(13) Geijtenbeek, T. B.; Van Vliet, S. J.; Koppel, E. A.; SanchezHernandez, M.; Vandenbroucke-Grauls, C. M.; Appelmelk, B.; Van Kooyk, Y. J. Exp. Med. 2003, 197 (1), 7-17.

(14) Appelmelk, B. J.; van Die, I.; van Vliet, S. J.; Vandenbroucke-Grauls, C. M.; Geijtenbeek, T. B.; van Kooyk, Y. J. Immunol. 2003, 170 (4), 1635-1639.

(15) Tacken, P. J.; de Vries, I. J.; Gijzen, K.; Joosten, B.; Wu, D.; Rother, R. P.; Faas, S. J.; Punt, C. J.; Torensma, R.; Adema, G. J.; Figdor, C. G. Blood 2005, 106 (4), 1278-1285.

(16) Kwon, D. S.; Gregorio, G.; Bitton, N.; Hendrickson, W. A.; Littman, D. R. Immunity 2002, 16 (1), 135-144.

(17) Grecco, H. E.; Lidke, K. A.; Heintzmann, R.; Lidke, D. S.; Spagnuolo, C.; Martinez, O. E.; Jares-Erijman, E. A.; Jovin, T. M. Microsc. Res. Tech. 2004, 65 (4-5), 169-179.

(18) Briggs, J. A.; Wilk, T.; Welker, R.; Krausslich, H. G.; Fuller, S. D EMBO J. 2003, 22 (7), 1707-1715.

(19) Yuasa, T.; Ishikawa, G.; Manabe, S.; Sekiguchi, S.; Takeuchi, K.; Miyamura, T. J. Gen. Virol. 1991, 72 (Pt 8), 2021-2024.

(20) Michalet, X.; Pinaud, F. F.; Bentolila, L. A.; Tsay, J. M.; Doose, S.; Li, J. J.; Sundaresan, G.; Wu, A. M.; Gambhir, S. S.; Weiss, S. Science 2005, 307 (5709), 538-544.

(21) Lidke, D. S.; Lidke, K. A.; Rieger, B.; Jovin, T. M.; Arndt-Jovin, D J. J. Cell Biol. 2005, 170 (4), 619-626.

(22) Lidke, D. S.; Nagy, P.; Heintzmann, R.; Arndt-Jovin, D. J.; Post, J N.; Grecco, H. E.; Jares-Erijman, E. A.; Jovin, T. M. Nat. Biotechnol. 2004, 22 (2), 198-203.

(23) Bergman, M. P.; Engering, A.; Smits, H. H.; van Vliet, S. J.; van Bodegraven, A. A.; Wirth, H. P.; Kapsenberg, M. L.; VandenbrouckeGrauls, C. M.; van Kooyk, Y.; Appelmelk, B. J. J. Exp. Med. 2004, 200 (8), 979-990.

(24) Pohlmann, S.; Baribaud, F.; Lee, B.; Leslie, G. J.; Sanchez, M. D.; Hiebenthal-Millow, K.; Munch, J.; Kirchhoff, F.; Doms, R. W. J. Virol. 2001, 75 (10), 4664-4672.

(25) Cambi, A.; de Lange, F.; van Maarseveen, N. M.; Nijhuis, M.; Joosten, B.; van Dijk, E. M.; de Bakker, B. I.; Fransen, J. A.; BoveeGeurts, P. H.; van Leeuwen, F. N.; Van Hulst, N. F.; Figdor, C. G. J. Cell Biol. 2004, 164 (1), 145-155. 
(26) Zerial, M.; McBride, H. Nat. Rev. Mol. Cell Biol. 2001, 2 (2), 107117

(27) Martinez, O.; Brackenridge, S.; El-Idrissi Mel, A.; Prabhakar, B. S. Int. Immunol. 2005, 17 (6), 769-778.

(28) Caparros, E.; Munoz, P.; Sierra-Filardi, E.; Serrano-Gomez, D.; PuigKroger, A.; Rodriguez-Fernandez, J. L.; Mellado, M.; Sancho, J.; Zubiaur, M.; Corbi, A. L. Blood 2006, 107 (10), 3950-3958.

(29) Nichols, B. J. Curr. Biol. 2003, 13 (8), 686-690.

(30) Rodal, S. K.; Skretting, G.; Garred, O.; Vilhardt, F.; van Deurs, B.; Sandvig, K. Mol. Biol. Cell 1999, 10 (4), 961-974.

(31) Mousavi, S. A.; Malerod, L.; Berg, T.; Kjeken, R. Biochem. J. 2004, 377 (Pt 1), 1-16.

(32) van Gisbergen, K. P.; Paessens, L. C.; Geijtenbeek, T. B.; van Kooyk, Y. Immunol. Lett. 2005, 97 (2), 199-208.
(33) Lidke, D. S.; Nagy, P.; Barisas, B. G.; Heintzmann, R.; Post, J. N.; Lidke, K. A.; Clayton, A. H.; Arndt-Jovin, D. J.; Jovin, T. M. Biochem. Soc. Trans. 2003, 31 (Pt 5), 1020-1027.

(34) Feinberg, H.; Mitchell, D. A.; Drickamer, K.; Weis, W. I. Science 2001, 294 (5549), 2163-2166.

(35) Bonifaz, L.; Bonnyay, D.; Mahnke, K.; Rivera, M.; Nussenzweig, M. C.; Steinman, R. M. J. Exp. Med. 2002, 196 (12), 1627-1638.

(36) Keler, T.; Ramakrishna, V.; Fanger, M. W. Expert Opin. Biol. Ther. 2004, 4 (12), 1953-1962.

(37) Gao, X.; Cui, Y.; Levenson, R. M.; Chung, L. W.; Nie, S. Nat. Biotechnol. 2004, 22 (8), 969-976.

NL0700503 